\title{
Reply to the Editor
}

\author{
Marcus J. Drake ${ }^{1} \cdot$ Kari Bo $^{2} \cdot$ Maria Zubieta $^{3}$
}

Received: 29 September 2016 / Accepted: 29 September 2016/Published online: 28 October 2016

(C) The International Urogynecological Association 2016

Urethral pressure profilometry (UPP) is a specialized test that focuses on a component of a complex functioning system. The two main parameters resulting from UPP are closure pressure and profile length, and these can provide diagnostic information about the clinical presentation of individual patients, but only in the context of all the indicators derived from clinical assessment. In our systematic review, we examined the published literature to determine whether UPP achieves one of the expectations of a diagnostic test, namely sensitivity to change after a therapeutic intervention. Overall, the literature showed that it is not sensitive when the treatment is pelvic floor muscle training (PFMT). There are several possible explanations for this;

- The test is insensitive

- The test was not done to necessary standards

- The treatment was not done well, because of either poor training by the supervising healthcare professional or poor adherence by the subject

This reply refers to the comment available at doi:10.1007/s00192-0163182-6.

Marcus J. Drake

marcus.drake@bui.ac.uk

1 School of Clinical Sciences, University of Bristol, Bristol, UK

2 Department of Sports Medicine, Norwegian School of Sport Sciences, Oslo, Norway

3 Bristol Urological Institute, Southmead Hospital, Bristol, UK
- PFMT does not act by affecting sphincter properties

- The research quality of the component studies was inadequate

In reviewing the literature [1], and from our own clinical experience, we feel that some women may experience improved sphincter function following PFMT, and this could result in improved UPP parameters. This is in accordance with the findings of Petros and Ulmsten [2]. Nonetheless, in the population overall, no systematic change in UPP parameters with PFMT has been shown. Accordingly, other explanations are required to explain the clinical efficacy of PFMT. Petros and Ulmsten suggest some possibilities, and there is a distinct chance that the functional results of PFMT vary and differ among individuals. If this is the case, the research needed to identify mechanisms of action of PFMT conclusively will need to be well-designed, with adequate sample sizes, and capturing a range of observations, each done to an adequate standard in welltaught patients demonstrated to be complying with the intervention.

\section{References}

1. Zubieta M, Carr RL, Drake MJ, Bo K. Influence of voluntary pelvic floor muscle contraction and pelvic floor muscle training on urethral closure pressures: a systematic literature review. Int Urogynecol J. 2016;27:687-696.

2. Petros PE, Ulmsten U. Role of the pelvic floor in bladder neck opening and closure I: muscle forces. Int J Urogynecol Pelvic Floor. 1997;8:74-80. 